\title{
Performance Evaluation of OSTBC Systems using different Channels
}

\author{
Amit Grover \\ Assistant Professor, ECE \\ SBSSTC, Ferozepur
}

\author{
Neeti Grover \\ Assistant Professor, DASH \\ SBSSTC, Ferozepur
}

\author{
Sanjolly Jain \\ Research Scholar (ECE) \\ SBSSTC, Ferozepur
}

\begin{abstract}
Due to the increased demand of wireless communication systems because of the features of the system which provides a wide coverage, high throughput and reliable services, the MIMO systems communication has come into existence and has been shown to be one of the most promising emerging wireless technologies that can efficiently boost the data transmission rate and capacity of wireless communication system.An effective and practical way to approach these demands of MIMO wireless channel is to employ Space Time (ST) Coding. The performance improvement of MIMO systems can be assessed by using diversity gain and spatial multiplexing gain. Space Time Coding transmits signal across the spatial and time domain simultaneously in order to achieve diversity gain without increasing bandwidth. In this paper, we exploit the space and time diversity to decode the quasi and rotated quasi orthogonal space time block codes (QOSTBC) based on Jakes model and Dent channel model. For Doppler shifting and Rayleigh distribution we make use of dent channel model. This provides fast decoding and gives better performance of communication system as compared to Jakes model. BER analysis has been presented in terms of diversity and code rate.
\end{abstract}

\section{Keywords}

Multi-input-multi-output (MIMO), Quasi Orthogonal SpaceTime Block codes (QOSTBC), rotated QOSTBC, Maximum Likelihood (ML) decoding.

\section{INTRODUCTION}

MIMO offers significant increases in data throughput and link range without additional bandwidth or transmit power. It achieves this by higher spectral efficiency and link reliability and or diversity. Because of these properties, MIMO is an important part of modern wireless communication [5].Communication in wireless channels is impaired predominantly by multipath fading. Multipath is the arrival of the transmitted signal at the receiver through differing angles and/or differing time delays and/or differing frequency [4]. One of the effective and practical ways to gain the capacity of the multiple inputs multiple outputs (MIMO) is to employ Space Time (ST) Coding which combines coding along with transmit diversity to achieve high diversity performance. It can be implemented in two forms ST-Trellis and ST-Block codes. The main problem with ST-Trellis scheme is that its decoding complexity increases exponentially with diversity and transmission rate. To address this problem Alamouti proposed Orthogonal [3] ST block codes (OSTBC) for $2 \times 1$ and $2 \times 2$ systems. These are modulation schemes that provide full diversity with simple coding and decoding technique. To overcome the shortcomings of orthogonal codes, quasi orthogonal codes of full rate have been proposed. The decoder performs pair wise symbol decoding [12] instead of single symbol decoding in these codes and these are called quasi orthogonal space time block codes [4] (QOSTBC) .Typically, quasi orthogonal space time codes perform best with ML decoding and provide full rate with maximum possible diversity. It is impossible to achieve full diversity if all the symbols are chosen from the same constellation's ,the solution to this problem is rotation based method, which aims at maximizing the minimum distance in the space time constellation by using different constellations for different transmitted symbols.

\section{MIMO CHANNEL MODEL}

By considering a communication system with ' $\mathrm{N}$ ' number of transmitting antennas and ' $M$ ' number of receiving antennas in an i. i. d Rayleigh Flat Fading channel, the performance of V-BLAST system can be accessed in correlated channel, by adopting a correlation-based channel model [12] which can be expressed as

$$
H \sim R_{R x}^{\frac{1}{2}} H_{w}\left(R_{T x}^{1 \nmid 2}\right)^{T}
$$

The fading effect is usually described statistically using the Rayleigh distribution. The amplitude of two quadrature Gaussian signals follows the Rayleigh distribution whereas the phase follows a uniform distribution [12].

\section{SPACE TIME BLOCK CODES (STBC)}

Space time block codes (STBCs) [3] have been proposed to realize the enhanced reliability of multi-antenna systems in which full diversity is achieved by using ML decoding [1] algorithm at the decoder. It is proved in [3] that a complex orthogonal design and corresponding Space Time Block code which provide full diversity and full transmission rate is not possible for more than two antennas. Full- rate orthogonal designs with complex elements in its transmission matrix are impossible for more than two transmit antennas [4].The only example of a full-rate full-diversity complex space-time block code using orthogonal designs is Alamouti schemes [4].The generator matrix [4] of Alamouti code can be obtained and to design the full rate, the codes with decoding pair of symbols [4] has been considered as shown in the equation 2 .

$$
G=\left(\begin{array}{rr}
\mathrm{G}\left(x_{1}, x_{2}\right) & G\left(x_{3}, x_{4}\right) \\
-G^{*}\left(x_{3}, x_{4}\right) & G^{*}\left(x_{1}, x_{2}\right)
\end{array}\right)
$$

To achieve code rate 1 and full diversity, for the complex orthogonal codes different constellations through different transmitted symbols are considered by rotating the symbols before transmission and it provide better performance as compared to QOSTBC.

\section{SYSTEM MODEL}

A Rayleigh fading channel constitutes Doppler' s spectrum is produced by synthesizing the complex sinusoids. The number 
of sinusoids to add must be large enough to approximate the Rayleigh amplitude. Furthermore, each of the sinusoidal generators must be weighted to generate the desired Doppler spectrum. This is the Jakes model that has been originally developed for the hardware simulation, but now frequently used in the software simulation.

Figure 1 illustrates the concept of jakes model. It has been assumed that all rays of the scattered components arriving in the uniform directions are approximated by $\mathrm{N}$ plane waves. Define $N 0=(N / 2-1) / 2$ where $\mathrm{N} / 2$ is limited to an odd number. Let us denote an angle of arrival for the nth plane wave, which is modeled as $\theta n=2 \pi n / N, n=1,2, N 0$. As shown in Figure 2, a sum of $N 0$ complex oscillator outputs with the frequencies of $w n=w d \cos \theta n, n=1,2, . N 0$, each corresponding to different Doppler shifts, is added to the output of a complex oscillator with a frequency of $w d=$ $2 \pi f m$.

$$
h(t)=\frac{E_{0}}{\sqrt{2 N_{0}+1}}\left\{h_{1}(t)+j h_{Q}(t)\right\}
$$

The real and imaginary parts, $\mathrm{h}(t)$ and $\mathrm{h} Q(t)$, in the total sum of the complex oscillators can be represented respectively as,

The real and imaginary parts [7], are given as,

$$
\begin{aligned}
& h(t)=2 \sum_{n=1}^{N_{0}}\left(\cos \phi_{n} \cos w_{n} t\right)+\sqrt{2} \cos \phi_{N} \cos w_{d} t \\
& h_{Q}(t)=2 \sum_{n=1}^{N_{0}}\left(\sin \phi_{n} \cos w_{n} t\right)+\sqrt{2} \sin \phi_{N} \cos w_{d} t
\end{aligned}
$$

Where, $\phi n$ and $\phi N$ are the initial phases of the n-th Dopplershifted sinusoid and the maximum Doppler frequency fm, respectively.

The initial phase is set to, $\quad \Phi_{\mathrm{N}}=0 \quad \phi n=\pi n N 0+1, n$ $=1,2, N 0$

The number of the Doppler-shifted sinusoids, N0, must be large enough to approximate the amplitude of the fading channel with a Rayleigh distribution.

$E E 0 \mathrm{~h} I(t) / \sqrt{ } 2 N 0+1) 2\}=E E 0 \mathrm{~h} Q(t) / \sqrt{ } 2 N 0+1) 2\}=E_{0}{ }^{2} / 2$ (6)

$$
\begin{aligned}
& \left\{\mathrm{h}^{2}(t)\right\}=E_{0}{ }^{2} \\
& \{\mathrm{~h}(t)\}=E_{0} \\
& \mathrm{E}\{\mathrm{h} I(t) \mathrm{h} Q(t)\}=0
\end{aligned}
$$

Equation (7) and Equation (8) confirm that the Jake model generates the fading signal with the average amplitude of $E_{0}$ and average energy of $E_{0}{ }^{2}$.Furthermore, Equation (6) and Equation (9) show that the real and imaginary parts of the channel are statistically independent with the average power of $E_{0}{ }^{2} / 2$

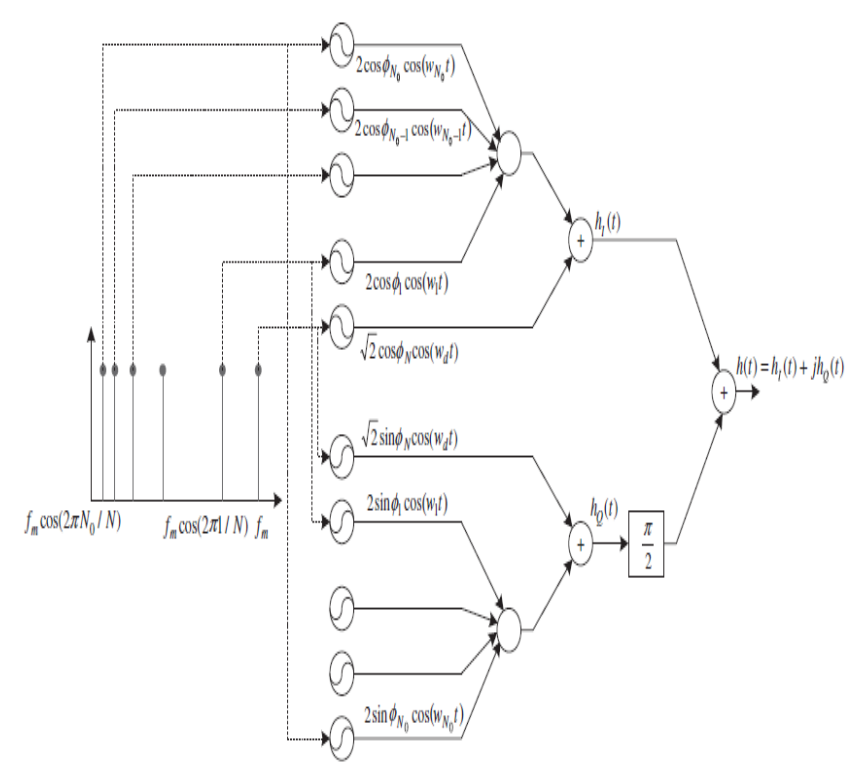

Figure 1 Implementation of Jakes model

The complex output of the jakes model [7], has been shown in equation (3) along with real and Imaginary defined in equation (4). Dent channel model [12] is a modification of Jake's model that removes the unwanted correlation of Jake's model.

At time $\mathrm{T}$, four elements in the $\mathrm{t}^{\text {th }}$ row of $\mathrm{C}$ are transmitted from the four transmit antenna. Since, the four given symbols are transmitted [12] in four time slots; this gives the code rate of 1 by using Maximum likelihood decoder.

\section{SIMULATION AND RESULTS}

The simulation parameters used throughout in this work are listed out in Table 1. Results are then plotted and discussed using these simulation parameters.

Simulation parameters of MIMO space time block coding system given in figure 1 .are listed in table 1 .

Table1. SIMULATION PARAMETERS FOR MIMO STBC

\begin{tabular}{llc}
\hline S. No. & \multicolumn{1}{c}{ Parameters } & Values \\
1 & No. of transmitters & 4 \\
2 & No. of receivers & 1 (Jakes) and 2 (Dent) \\
& & \\
3 & Max. Doppler shift(fm) & $200 \mathrm{~Hz}$ \\
4 & Sampling frequency(fs) & $8000 \mathrm{~Hz}$ \\
5 & Career modulation & BPSK,QPSK,16QAM \\
6 & Bandwidth & $20 \mathrm{MHz}$ \\
7 & Sampling time(ts) & $1 / \mathrm{fs}$ \\
8 & No. of Doppler shift(N) & 8 \\
\hline
\end{tabular}


The simulation result is conducted in MATLAB. In this we will make comparison of performance of QOSTBC with rotated QOSTBC using Dent model and Jakes model. Results with different modulation techniques are plotted for BER with SNR. The modulation technique used is BPSK, QPSK and 16QAM with rotation of $\Pi / 2, \Pi / 4$ and $\Pi / 4$ respectively for transmission of 1.5 bits $/ \mathrm{s} / \mathrm{Hz}$. Figure.2 (a) and Figure.3 (a), shows better BER performance with BPSK as compared to QPSK and 16-QAM. Figure.4 (a) shows the comparison of quasi and rotated quasi OSTBC with BPSK modulation using Dent model in which it is clearly observed that rotated quasi OSTBC gives better result when compared to quasi OSTBC. BER performance is better using BPSK as compared to QPSK and 16-QAM. Figure 2(a) shows that quasi OSTBC with BPSK modulation gives better result. At SNR $21 \mathrm{~dB}$, BER is reduced to 0.0040 , as it is clear from the figure by employing 4 transmitting antennas the system performance is enhanced in Jakes Model. From Table.2, At SNR=12dB, 4X2 antenna configuration BPSK modulation has minimum (0.0019) BER than another modulation schemes like QPSK, 16-QAM in Quasi- OSTBC MIMO System in Dent Channel Model. So we can say that in this configuration BPSK modulation gives better BER performance.

Table2. Simulation Parameters for Quasi-OSTBC MIMO STBC System using Dent Model

\begin{tabular}{|l|l|}
\hline Modulation & BER \\
\hline BPSK & .0019 \\
\hline QPSK & 0.0032 \\
\hline $16-$ QAM & \\
\hline & 0.0158 \\
\hline
\end{tabular}

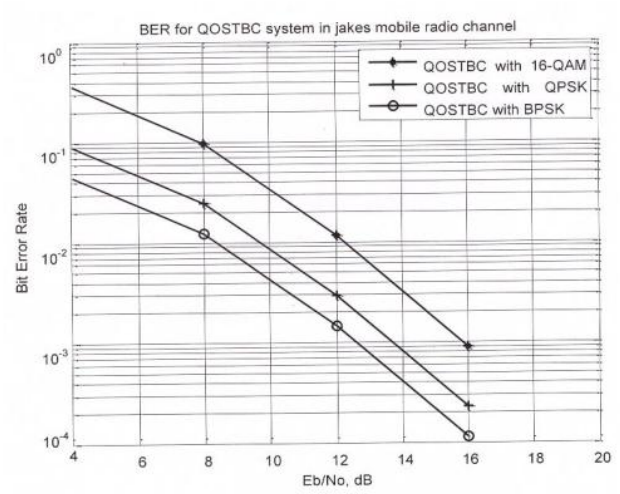

BER for Quasi-OSTBC system

Figure 2 (a)

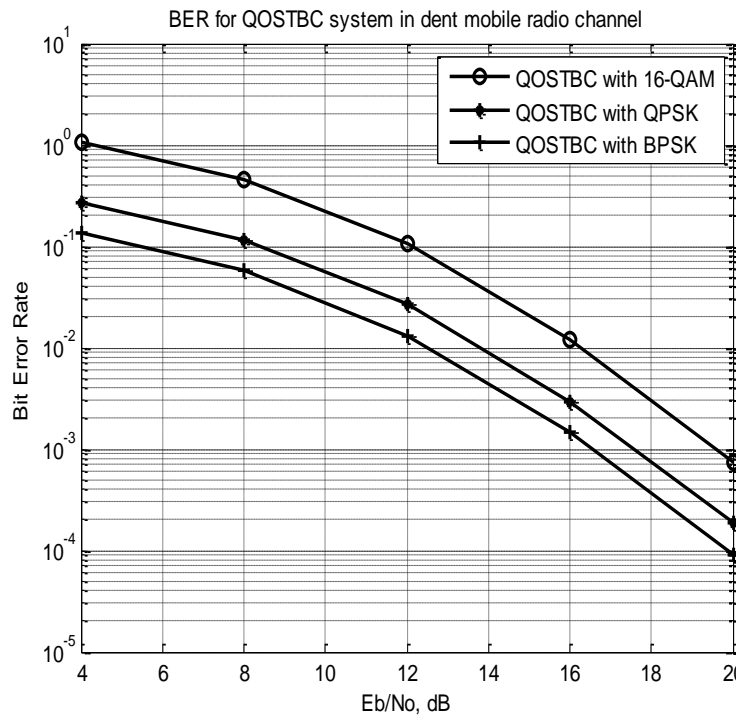

Figure 2 (b)

Figure2. BER for Quasi-OSTBC system with Jakes model and Dent channel model

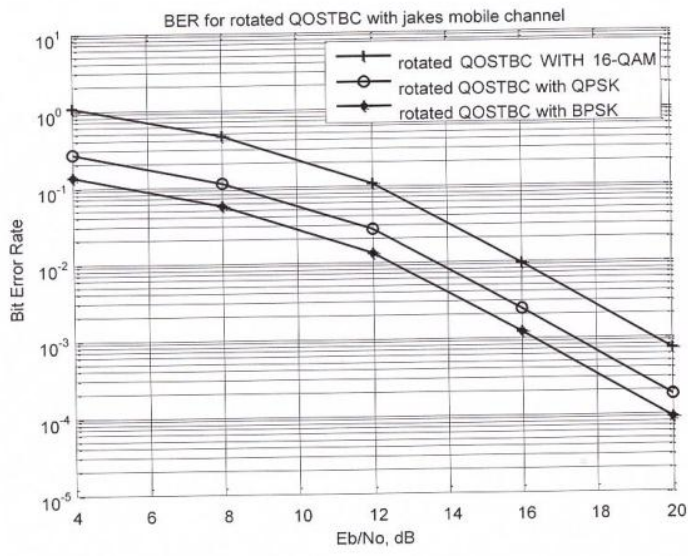

BER for rotated quasi OSTBC system

Figure 3 (a)

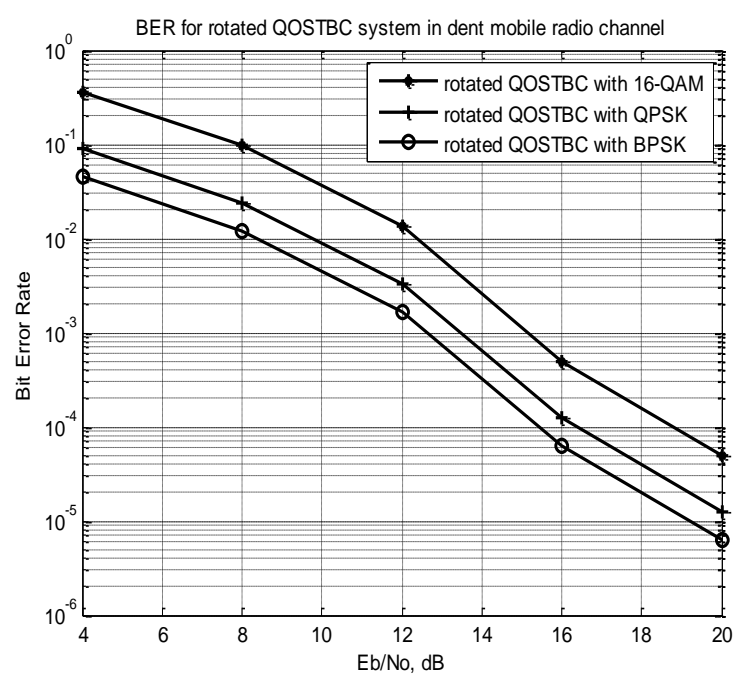

Figure 3 (b) 
Figure3. BER for rotated quasi-OSTBC system with Jakes model and Dent channel model

BER performance is better using BPSK as compared to QPSK and 16-QAM. Figure 3(a) shows that rotated quasi OSTBC with BPSK modulation gives better result. At SNR $20 \mathrm{~dB}$, BER is reduced to 0.004 , as it is clear from the figure by employing 4 transmitting antennas the system performance is enhanced in Jakes Model. From Table.3, at SNR=12dB, 4X2 antenna configuration BPSK modulation has minimum (0.000003162) BER than another modulation schemes like QPSK, 16-QAM. in rotated Quasi- OSTBC MIMO System in Dent Channel Model. So we can say that in this configuration BPSK modulation gives better BER performance and by using 16-QAM modulation technique Rotated Quasi - OSTBC gives worst result.

Table3. Simulation Parameters for Rotated Quasi-

OSTBC MIMO STBC System using Dent Model

\begin{tabular}{|l|l|}
\hline \multicolumn{1}{|c|}{ Modulation } & BER \\
\hline BPSK & .000003162 \\
\hline QPSK & 0.0001 \\
\hline 16-QAM & 0.000063 \\
\hline
\end{tabular}

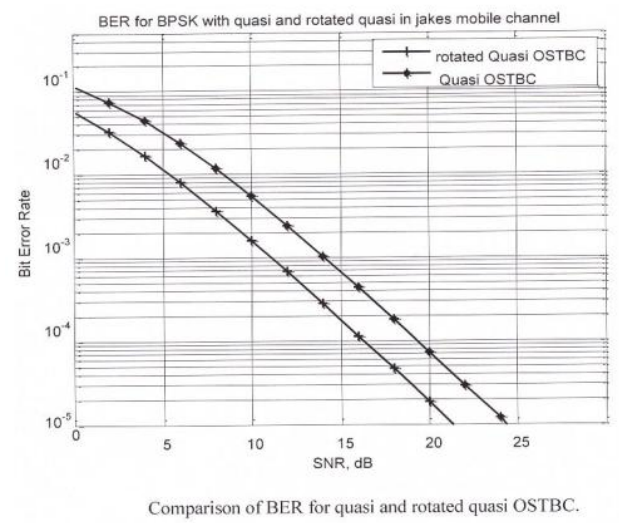

Figure 4 (a)

BER performance is better using BPSK as compared to QPSK and 16-QAM. Figure 4(a) shows that quasi OSTBC with BPSK modulation gives better result. At SNR $21 \mathrm{~dB}$, BER is reduced to 0.0040 . It has been clearly observed that rotated quasi OSTBC gives better results when compared to quasi OSTBC in Jakes Model. From Table.4, At SNR=15dB, 4X2 antenna configuration, for Rotated Quasi OSTBC BER is 0.000063 and for Quasi-OSTBC MIMO System BER is 0.0000251 in BPSK modulation. So we can say that Rotated Quasi OSTBC has better performance than Quasi-OSTBC MIMO System.

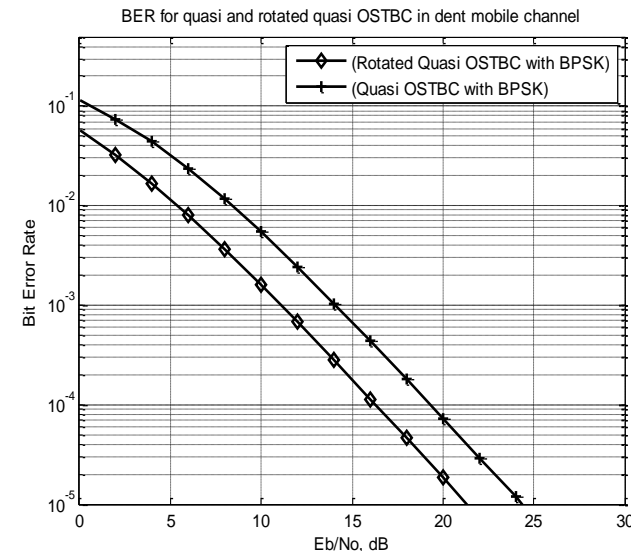

Figure 4 (b)

Figure4. BER for rotated quasi and quasi OSTBC system with Jakes model and Dent channel model

Table4. Simulation Parameters for comparison between Rotated Quasi-OSTBC and Quasi-OSTBC MIMO System using BPSK Modulation in Dent Model.

\begin{tabular}{|l|l|}
\hline Modulation & BER \\
\hline Rotated Quasi-OSTBC & .00000632 \\
\hline Quasi-OSTBC & 0.0000251 \\
\hline
\end{tabular}

\section{CONCLUSIONS}

In this paper, the performance of MIMO systems under mobile radio channel using Jakes Model and Dent model has been investigated. Further, system performance has been compared with three different modulation techniques and the system with BPSK modulation gives better results. Quasi orthogonal space time block coding provide code rate of 1 and rotated quasi orthogonal space time block coding provide full rate and full diversity. By using ML decoder, the decoding complexity of the system has been reduced and the performance of the system has been enhanced. Finally, It has been observed that the system performance enhances using Dent channel model.

\section{REFERENCES}

[1] Bhasker gupta and Davinder S.Saini (2011), "BER Analysis of Space-Frequency Block Coded MIMOOFDM Systems Using Different Equalizers in QuasiStatic Mobile Radio Channel" Proc. of International Conference on Communication Systems and Network Technologies (CSNT-11), pp. 520-524.

[2] Flores, J.; Sánchez, J.; Jafarkhani, H.; (2010) , "Differential Quasi-Orthogonal Space-Frequency Trellis Codes," IEEE Trans. Wireless Commun., vol.9, no.12, pp.3620-3624.

[3] V. Tarokh, H. Jafarkhani and A.R. Calderbank (1999),"Space Time Block Codes from orthogonal designs", IEEE Trans. Inform. Theory, Vol.45, pp. 14561467. 
[4] Hamid Jafarkhani(2001),"A Quasi-Orthogonal Space Time Block Codes", IEEE Transaction on Communications, vol.49, no.1.

[5] S.M. Alamouti (1998),"A Simple Transmitter diversity scheme for wireless communications", IEEE J.Select. Areas Commun, vol.16, pp. 1451-1458.

[6] Andreas A. Huttera, Selim Mekrazib, Beza N. Getuc and Fanny Platbrooda (2005), "Alamouti-Based SpaceFrequency Coding for OFDM," in Springer journal of wireless personal communications, vol. 35 , no. 1-2, pp. 173-185.

[7] Yong Soo Cho, Jaek won Kim, Won Young Yang, Chung Gu Kang (2010), "MIMO-OFDM Wireless Communications with MATLAB", 1st edition, John Wiley \& Sons (Asia) Pte Ltd.

[8] M. Rezk and B. Friedlander (2011)," On High Performance MIMO Communications with Imperfect Channel Knowledge" IEEE Trans. Wireless Commun., vol. 10, no. 2, pp. 602-613.

[9] V. Tarokh, H. Jafarkhani and A.R. Calderbank (1999),"Space Time Block Coding for wireless communications: Performance results", IEEE J. Select. Areas Commun, vol.17, pp. 451-460.

[10] A.V. Geramite and J. Seberry, Orthogonal Designs, Quadratic forms and Hadamard Matrices (1979),Ser. Lecture Notes in Pure and Applied Mathematics. New York: Marcel Dekkar,vol.43.

[11] Wei Xiang, Julian Russell and Yafeng Wang (2010),"” ICI Reduction Through Shaped OFDM in Coded MIMO - OFDM Systems", International Journal on Advances in Telecommunications, vol.3, no 3 \& 4. http://www.iariajournals.org/telecommunications/

[12] Manbir Kaur, Amit Grover (2012), "Study and Simulation of Quasi and Rotated Quasi Space Time Block Codes in MIMO systems using Dent Channel model", Innovative System Design and Engineering, vol. 3, no 10 , pp.72-81.

\section{AUTHORS' PROFILE}

Amit Grover (M'06-SM'09-PI'11\&12) The author became a Member (M) of Association ISTE in 2006, a Senior Member (SM) of society SELCOME in September 2009, and a ProjectIn charge (PI) in august 2011 and in September 2012. The author place of birth is Ferozepur, Punjab, India on $27^{\text {th }}$, September 1980.The author received his M. Tech degree in Electronics and Communication Engineering from Punjab Technical University, Kapurthla, Punjab, India in 2008 and received his B. Tech degree in Electronics and Communication Engineering from Punjab Technical University, Kapurthala, Punjab, India in 2001. Currently, he is working as an Assistant Professor in Shaheed Bhagat Singh State Technical Campus, Ferozepur, Punjab, India. The author is a Reviewer of many International Journals. His area of interest includes signal processing, MIMO systems, Wireless mobile communication, High speed digital communications and 4G Wireless Communications.

Neeti Grover received her master degree in Applied Sciences from Guru Nanak Dev University, Amritsar, and Punjab, India in 2007 and received her Bachelor' s degree from Guru Nanak Dev University, Amritsar, Punjab, India in 2004. Her place of birth is Jallandhar, Punjab, India on $29^{\text {th }}$, December 1983. Currently, she is working as an Assistant Professor in the department of Applied Sciences and Humanities in Shaheed Bhagat Singh State Technical Campus (Poly Wing), Ferozpur, Punjab, India.

The author received her B. Tech degree in Electronics and Communication Engineering in 2012 from North West Institute of Engineering and Technology, Affiliated to Punjab Technical University, Kapurthjala, Punjab, India. Her place of birth is Ferozepur, Punjab, India on $22^{\text {nd }}$, December 1990. At present She has been doing her research work under the guidance of Mr. Amit Grover, Assistant Professor, Department of ECE, Shaheed Bhagat Singh State Technical Campus, Moga road, Ferozepur-152004, Punjab, India. 